\title{
Tolvaptan and its potential in the treatment of hyponatremia
}

\section{Megan B Dixon \\ Y Howard Lien}

Department of Medicine, University of Arizona, Arizona Kidney Disease and Hypertension Center, Tucson, Arizona, USA
Correspondence: $Y$ Howard Lien 45 I I N. Campbell Ave, Suite 100 , Tucson AZ 857I8, USA

Tel: + I 5205296500

Fax: + I 5202097337

Email lienhoward@gmail.com

\begin{abstract}
Tolvaptan is a selective arginine vasopressin (AVP) $V_{2}$ receptor blocker used to induce free water diuresis in the treatment of euvolemic or hypervolemic hyponatremia. Currently the orally active medication is in the final stages prior to approval by the FDA for outpatient therapy. It appears to be safe and effective at promoting aquaresis and raising serum sodium levels in both short- and long-term studies. Tolvaptan is also effective for treatment of congestive heart failure (CHF) exacerbation, but whether there are long standing beneficial effects on CHF is still controversial. Prolonged use of tolvaptan leads to increased endogenous levels of AVP and perhaps over-stimulation of $\mathrm{V}_{1 \mathrm{~A}}$ receptors. Theoretically this activation could lead to increased afterload and cardiac myocyte fibrosis, causing progression of CHF. However, after 52 weeks of tolvaptan therapy there was no worsening of left ventricular dilatation. In addition, tolvaptan is metabolized by the CYP3A4 system; thus physicians should be aware of the potential for increased interactions with other medications. Tolvaptan is a breakthrough in the therapy of hyponatremia as it directly combats elevated AVP levels associated with the syndrome of inappropriate secretion of antidiuretic hormone, congestive heart failure, and cirrhosis of the liver.
\end{abstract}

Keywords: hyponatremia, arginine vasopressin, vasopressin receptors, syndrome of inappropriate antidiuretic hormone, congestive heart failure, liver cirrhosis

\section{Introduction}

Volume overload and hyponatremia cause significant morbidity and may lead to increased mortality in patients with the syndrome of inappropriate antidiuretic hormone (SIADH), congestive heart failure (CHF), and liver cirrhosis (Lee et al 2003). Elevated levels of arginine vasopressin (AVP or antidiuretic hormone) contribute to this state of total body water excess and hyponatremia (Goldsmith et al 1983). Diuretics and free water restriction have historically been used to combat this condition but have unwanted side effects including electrolyte imbalances, arrhythmias, and renal failure. Vasopressin receptor antagonists (VRAs or "vaptans") directly target stimulated AVP receptors in the collecting duct of the kidney, down-regulating aquaporin insertion and free water absorption. Tolvaptan, an oral $\mathrm{V}_{2}$-receptor specific antagonist, has been very effective and safe in outpatient trials in treating hyponatremia and volume overload. It was recently approved by the EMEA (European Medicines Agency) for use in Europe. The US FDA has recommended tolvaptan for the treatment of hyponatremia, but it is currently not approved for use in the US. Presently, only intravenous conivaptan has been approved by the FDA for the short-term treatment (4 days) of hyponatremia in hospitalized patients. Unfortunately, the lack of follow-up therapy significantly limits the usage of conivaptan. An efficacious oral agent would be ideal for the outpatient management of chronic hyponatremia.

\section{Regulation of vasopressin}

AVP or antidiuretic hormone $(\mathrm{ADH})$ is produced in the hypothalamus and delivered to the posterior pituitary for release into systemic circulation. Secretion of AVP is 
mediated by several mechanisms. Osmotic pressure is the most sensitive stimulus for AVP release and is mediated by osmoreceptors in the hypothalmus. Sodium concentration greatly influences osmotic pressure. A decrease in osmolality as minimal as $1 \%$ to $2 \%$ rapidly suppresses AVP secretion and induces aquaresis. Arterial pressure reduction also stimulates AVP release, but typically there must be a significant reduction of $10 \%$ to $20 \%$ as sensed by baroreceptors in the left atrium and aorta. It appears that arterial pressure provokes AVP release by lowering the set point of the osmoregulatory system (Schrier 2007). Heart failure and cirrhosis cause an arterial underfilling, stimulating AVP release, and overriding hypo-osmolality-induced AVP inhibition (Lien and Shapiro 2007). The result is hyponatremia in the face of total body volume overload. As for SIADH, the excessive release of vasopressin is independent of osmolality and volume status. Patients exhibit mild volume overload due to water retention and significant hyponatremia as a consequence.

\section{Vasopressin receptors}

AVP stimulates both $\mathrm{V}_{1 \mathrm{~A}}$ and $\mathrm{V}_{2}$ receptors. $\mathrm{V}_{1 \mathrm{~A}}$ receptors are located in vascular smooth muscle and cause arterial vasoconstriction to compensate for low arterial pressure. AVP exerts its anti-diuretic effect by stimulating the $\mathrm{V}_{2}$ receptor located on the basolateral side of the principal cell in the cortical collecting duct. AVP $V_{2}$ is a $\mathrm{G}$ proteincoupled receptor, which when stimulated initiates adenylate cyclase and leads to increased intracellular cAMP (Lien and Shapiro 2007). Elevated cAMP signals placement of vesicle-encased aquaporin-2 channels in the principal cell apical membrane, facilitating free water absorption in the collecting tubule. Most of the "vaptans," including tolvaptan, are $\mathrm{V}_{2}$ receptor-selective, except conivaptan, which blocks both receptors.

\section{Pharmacological actions of tolvaptan}

Tolvaptan (study name OPC-41061) is an orally active, non-peptide, selective $\mathrm{V}_{2}$ receptor antagonist that blocks AVP binding and promotes free water excretion. The comparisons and interactions between VRAs and diuretics are clinically important because diuretics are likely to be used in conjunction with tolvaptan. Shoaf et al (2007) performed a pilot, randomized, cross-over study to determine the pharmacologic interactions between tolvaptan and furosemide or hydroclorothiazide (HCTZ) (Shoaf et al 2007). Twelve healthy young Caucasian men were randomized to receive $30 \mathrm{mg}$ of tolvaptan, then $80 \mathrm{mg}$ of furosemide (6) or
$100 \mathrm{mg}$ of HCTZ (6), and finally both medications together. A 48-hour washout period was observed between therapies. Multiple measurements were gathered including urinary output, electrolyte concentrations of plasma and urine, and neurohormone levels in addition to pharmacological parameters. Tolvaptan alone had a 50\% increase in 24-hour urine output compared with furosemide and HCTZ (dosed once daily). Urinary sodium was $50 \%$ greater during furosemide and HCTZ therapy in comparison to tolvaptan. Kaluresis did not differ largely between drugs. Plasma levels of sodium increased during therapy with tolvaptan and decreased with furosemide and HCTZ. Renin activity was increased at 24 hours post dose for all patients treated with furosemide, HCTZ, or a combination of either with tolvaptan. Tolvaptan alone had negligible increases in renin activity but did show increased AVP levels at 24 hours. Despite increased AVP levels, blood pressure did not differ in patients treated with tolvaptan. Interestingly, the maximum tolvaptan concentration $\left(\mathrm{C}_{\max }\right)$ and area under the plasma concentration-time curve $\left(\mathrm{AUC}_{\infty}\right)$ was increased for tolvaptan plus furosemide, but urinary and hemodynamic parameters were unchanged. There was no additive effect with diuretic adjunct therapy in this study.

\section{Tolvaptan for treating hyponatremia}

SALT 1 and 2 (Study of Ascending Levels of Tolvaptan in Hyponatremia) were designed to focus specifically on changes in serum sodium in patients with hyponatremia from multiple disorders including SIADH, heart failure and cirrhosis (Shrier et al 2006). Two separate randomized, double-blind, placebo-controlled trials of 200 to 250 patients were performed to determine reproducibility. Patients were randomized to tolvaptan treatment (with dose adjustment from 15 to $60 \mathrm{mg}$ as needed) versus placebo and treated for 30 days with follow-up assessment at 1 week post completion. The area-under-the-curve (AUC) for increase in sodium levels from baseline to day 4 and day 30 was significantly greater in the tolvaptan versus placebo treatment group $(\mathrm{p}<0.001)$. The average baseline serum sodium in both groups was $128 \mathrm{mmol} / \mathrm{L}$. At day 4, tolvaptantreated patients had an average sodium of 134 and placebo $130 \mathrm{mmol} / \mathrm{L}(\mathrm{p}<0.001)$. Serum sodium rose to an average of $136 \mathrm{mmol} / \mathrm{L}$ in the tolvaptan group at day 30, but was unchanged in the placebo group. The greatest difference was in patients with marked hyponatremia. Side effects were minimal and included dry mouth and increased thirst. Overcorrection of hyponatremia $(>0.5 \mathrm{mmol} / \mathrm{L} / \mathrm{h})$ occurred in less than $2 \%$ of tolvaptan-treated patients, yet none 
of them developed central pontine myelinolysis. Once tolvaptan was discontinued at day 30 , serum sodium levels returned to similar levels or below those of their placebo counterparts.

Tolvaptan therapy versus fluid restriction for the treatment of hyponatremia was studied by Gheorghiade et al (2006). Twenty-eight hospitalized subjects with a serum sodium $<135 \mathrm{mmol} / \mathrm{L}$ were randomized to tolvaptan alone (17) versus fluid restriction of $1200 \mathrm{~mL} /$ day (11) for 27 days. Tolvaptan doses were increased from 15 to $60 \mathrm{mg}$ /day as needed to increase serum sodium levels. The primary endpoint was normalization of serum sodium $(>135 \mathrm{mmol} / \mathrm{L})$ or an increase $\geq 10 \%$ from baseline. Normalization of serum sodium occurred in $50 \%$ of patients by day 4 when treated with tolvaptan and by day 8 on fluid restriction $(\mathrm{p}<0.03)$. Serum sodium increased $5.7 \pm 3.2 \mathrm{mmol} / \mathrm{L}$ in the tolvaptantreated group and $1.0 \pm 4.7 \mathrm{mmol} / \mathrm{L}$ in the fluid-restriction group ( $\mathrm{p}=0.0065)$. Clearly tolvaptan showed superiority in treating hyponatremia compared with fluid restriction alone. As patients are likely to be non-compliant with fluid restriction, tolvaptan is likely to be very successful in outpatients.

\section{Effects of tolvaptan on congestive heart failure: short-term effects}

Congestive heart failure is a common cause of hyponatremia and associated with elevated AVP levels (Goldsmith et al 1983). Tolvaptan has largely been studied in the setting of $\mathrm{CHF}$ as a novel way to treat $\mathrm{CHF}$ exacerbations and perhaps alter the natural course of heart failure. Three major studies that investigated short-term effects of tolvaptan on $\mathrm{CHF}$ and related hyponatremia are summarized in Table 1.

Gheorghiade et al (2003) reported the first randomized, double-blinded trial of tolvaptan in patients with symptomatic heart failure. In this study, 254 patients with NYHA class I, II or III CHF were randomly assigned to receive tolvaptan 30 , 45 or $60 \mathrm{mg}$ versus placebo for 25 days. Patients were kept on stable doses of CHF ameliorating medications including angiotensin-converting enzyme inhibitors/angiotensin receptor blockers (ACE-I/ARB), beta-blockers, and furosemide and allowed to drink fluids freely. Weight loss was approximately $-0.8 \mathrm{~kg}$ for patients treated with tolvaptan (regardless of dose) versus a gain of $+0.3 \mathrm{~kg}$ for placebo at day 1 ( $\mathrm{p}<0.001$ for all groups and placebo). Patients maintained this weight reduction at day 25 but did not lose further weight while on tolvaptan. Significant aquaresis was noted in the tolvaptan groups in comparison to placebo. Urine output measured approximately $4 \mathrm{~L}$ /day on therapy with tolvaptan and only 2 L/day with placebo, while urine osmolality decreased in tolvaptan groups and increased in the placebo group. Hyponatremic enrollees had larger increases in serum sodium than normonatremic counterparts. Eighty-two percent of the hyponatremic tolvaptan enrollees attained a normal

Table I Short-term effects of tolvaptan on congestive heart failure

\begin{tabular}{|c|c|c|c|c|c|c|c|}
\hline \multirow[t]{2}{*}{ Study } & \multirow[t]{2}{*}{ Treatment } & Weight loss (kg) & Weight loss (kg) & $\begin{array}{l}\text { Urine output } \\
\text { (L/day) }\end{array}$ & \multirow{2}{*}{$\begin{array}{l}\text { Urine } \\
\text { osmolality } \\
\text { (mOsm/kg) } \\
\text { Day I }\end{array}$} & \multirow{2}{*}{$\begin{array}{l}\text { Change } \\
\text { in Sna } \\
\text { (meq/L) } \\
\text { Day I }\end{array}$} & \multirow{2}{*}{$\begin{array}{l}\text { Change in } \\
\text { Sna (meq/L) } \\
\text { Day } 7\end{array}$} \\
\hline & & Day I & Day 7 & Day I & & & \\
\hline Tolvaptan in & Tolvaptan & & & & & & \\
\hline \multirow[t]{4}{*}{ patients with $\mathrm{CHF}$} & $30 \mathrm{mg}$ & $0.79 \pm 0.99$ & & $3.9 \pm 0.6$ & $15.5 \pm 190$ & & \\
\hline & $45 \mathrm{mg}$ & $0.96 \pm 0.93$ & & $4.2 \pm 0.9$ & $52.4 \pm 165$ & & \\
\hline & $60 \mathrm{mg}$ & $0.84 \pm 0.02$ & & $4.6 \pm 0.4$ & $118.8 \pm 132$ & & \\
\hline & Placebo & $+0.32 \pm 0.46$ & & $2.3 \pm 0.2$ & $+135.8 \pm 189$ & & \\
\hline \multirow[t]{5}{*}{ ACTIV in CHF } & Tolvaptan & & & & & & \\
\hline & $30 \mathrm{mg}$ & $1.80(-3.85$ to -0.5$)$ & & & & +2.77 & \\
\hline & $60 \mathrm{mg}$ & $2.10(-3.1$ to -0.85$)$ & & & & +3.38 & \\
\hline & $90 \mathrm{mg}$ & $2.05(-2.80$ to -0.6$)$ & & & & +3.50 & \\
\hline & Placebo & $0.60(-1.60$ to 0$)$ & & & & 0.20 & \\
\hline \multirow[t]{6}{*}{ EVEREST } & Tolvaptan & & & & & & \\
\hline & $30 \mathrm{mg}$ & & & & & & \\
\hline & Trial A & $1.71 \pm 1.8$ & $3.35 \pm 3.3$ & & & +3.28 & +2.07 \\
\hline & Trial B & $1.82 \pm 2.01$ & $3.77 \pm 3.6$ & & & & \\
\hline & Placebo A & $0.99 \pm 1.83$ & $2.73 \pm 3.3$ & & & 0.41 & 1.06 \\
\hline & Placebo B & $0.95 \pm 1.85$ & $2.79 \pm 3.5$ & & & & \\
\hline
\end{tabular}

All above comparisons of tolvaptan versus placebo are statistically significant $(p<0.05)$. 
serum sodium level by the end of the 25 days. Although not statistically significant, AVP concentrations were higher in tolvaptan-treated patients than placebo. Again, there were no differences in blood pressure measurements between both groups.

Gheorghiade et al (2004) then launched a larger, randomized trial of tolvaptan in hospitalized patients with congestive failure exacerbation, the ACTIV in CHF investigation (Acute and Chronic Therapeutic Impact of a Vasopressin antagonist in Congestive Heart Failure). Three hundred nineteen patients with reduced ejection fraction $(<40 \%)$ and persistence of congestive symptoms despite standard therapy with diuretics were randomized to tolvaptatn $30 \mathrm{mg}, 60 \mathrm{mg}, 90 \mathrm{mg}$ daily, or placebo for up to 60 days. Patients were continued on other CHF therapy including diuretics, ACE-I/ARB, and beta-blockers. The acute primary outcome was change in body weight at 24 hours. Tolvaptan showed significant decreases in mean body weight (regardless of dose) in comparison to placebo. Serum sodium increased by approximately $3 \mathrm{meq} / \mathrm{L}$ for tolvaptan-treated patients and decreased by $-0.2 \mathrm{meq} / \mathrm{L}$ in the placebo group. Patients with underlying hyponatremia that were treated with tolvaptan often had a normalization of their serum sodium levels. At time of discharge, dyspnea scores were significantly improved in the tolvaptan treatment $\operatorname{arm}(\mathrm{p}=0.04)$.

The third study was the EVEREST trial (Efficacy of Vasopressin antagonism in Heart Failure Outcome Study with Tolvaptan), which also examined short- and long-term effects of tolvaptan therapy in patients with $\mathrm{CHF}$ exacerbation (Cheorghiade et al 2007). Two identical randomized, double-blinded, placebo-controlled trials (A and B) of $2000+$ patients with worsening heart failure symptoms were performed in North America, South America, and Europe. Patients were given appropriate therapy with diuretics, ACE-I/ARB, beta-blocker at the discretion of investigators and then randomized to receive $30 \mathrm{mg}$ of tolvaptan versus placebo. Primary outcomes included body weight at day 7 or discharge (whichever came first) and changes in clinical status measured with a visual analog scale. Weight reduction was significantly reduced in patients treated with tolvaptan versus placebo at day 1 and day 7 or discharge $(p<0.001$ for all comparisons). Secondary outcomes, including self-reported dyspnea at day 1 and lower extremity edema evaluated by clinicians, were improved on tolvaptan $(\mathrm{p}<0.05$ except edema evaluation in Trial A). Serum sodium elevated a mean of $3.3 \mathrm{meq} / \mathrm{L}$ at day 1 and remained elevated $2.0 \mathrm{meq} / \mathrm{L}$ at day 7 or discharge in tolvaptan-treated groups and dropped in placebo groups $(\mathrm{p}<0.001)$. The change in serum sodium was most impressive in patients with a baseline serum sodium $<134 \mathrm{meq} / \mathrm{L}$ at randomization. For this group, those receiving tolvaptan had a serum sodium increase of $5.5 \mathrm{meq} / \mathrm{L}$ at day 7 or discharge, while those of placebo had an elevation of $1.9 \mathrm{meq} / \mathrm{L}(\mathrm{p}<0.001)$ (Konstam et al 2007). Despite significantly more thirst and dry mouth in the tolvaptan groups there were no other differences in adverse effects. No increased incidence of hypotension, arrhythmia, renal failure, or electrolyte abnormality was noted in tolvaptan-treated patients. The short-term arm of EVEREST study complemented the results shown 3 years earlier in ACTIV in CHF.

The short-term effects of tolvaptan on renal hemodynamics in $\mathrm{CHF}$ patients have been compared with furosemide and placebo by Costello-Boerrigter et al (2005). The randomized, placebo-controlled crossover study investigated the changes in urine output, sodium and potassium excretion, renal blood flow (RBF), and glomerular filtration rate (GFR) in 14 patients with stable class II or III heart failure and ejection fraction $<40 \%$. Patients received either tolvaptan $30 \mathrm{mg}$ or placebo on day 1 and then were crossed to receive the other medication on day 3 . Both groups then received $80 \mathrm{mg}$ of furosemide on day 5 for comparison. All other CHF medications including maintenance ACE-I/ARB, beta-blockers, aspirin, and diuretics were held prior to the start of the study. Patients were maintained on similar sodium-restricted diets and were given water at prescribed points during the day. No raw data were available in the study text, but analysis noting statistical significance was reported. Urine output increased significantly with tolvaptan and furosemide treatment versus placebo, but there was no advantage between the two. Urinary sodium and potassium excretion was statistically greater in furosemide than in tolvaptan and placebo treatment. Urine osmolality was lowest in tolvaptan, significantly reduced from placebo $(p<0.05)$ but not from furosemide treatment $(\mathrm{p}=0.10)$. Renal blood flow, measured by PAH clearance, was significantly lower in furosemide therapy than tolvaptan and placebo. Renal blood flow actually was mildly increased from placebo $(\mathrm{p}=0.19)$ in tolvaptan treatement. Finally, GFR (measured by inulin clearance) was similar between all three therapies. Neurohormonal measurements at 0.5 and 2.5 hours after medication administration were also attained - AVP, renin, aldosterone, atrial natriuretic peptide, beta natriuretic peptide, and norepinephrine. AVP levels were similar among the three treatment groups (likely related to short duration of tolvaptan therapy), with only a significant increase in plasma renin activity in furosemide versus placebo but not furosemide versus tolvaptan. The authors concluded 
that tolvaptan was a safe aquaretic similar in efficacy to furosemide therapy for mild to moderate CHF.

In summary, aquaresis and elevations in serum sodium, especially in hyponatremic patients, were well-documented in short-term investigations. There was no dose-related improvement in weight loss, serum sodium increase, or CHF symptoms with escalating doses of tolvaptan. For that reason, a single dose of $30 \mathrm{mg}$ daily was used for many subsequent studies.

\section{Effects of tolvaptan on congestive heart failure: long-term effects}

Long-term effects of tolvaptan on morbidity and mortality associated with $\mathrm{CHF}$ have not been validated. As mentioned earlier, both the ACTIV in CHF and the EVEREST studies examined long-term outcomes. In a third study, by Udelson et al (2005), left ventricular end diastolic volume (LVEDV) and other parameters were measured while on tolvaptan. The results of these three studies are summarized in Table 2.

In the ACTIV in CHF study, the long-term primary outcome (60 days) was worsening CHF, defined as death, CHF-related hospitalization, or unscheduled physician visit. There were no differences in worsening heart failure between the tolvaptan groups versus placebo. Tolvaptan-treated patients tended to require less maintenance diuretic than their placebo counterparts. A post hoc analysis identified 2 groups with improved 60-day mortality on tolvaptan in comparison with placebo. These groups were patients with elevated blood urea nitrogen levels $(p=0.07)$ and severe congestive symptoms $(\mathrm{p}=0.03)$. Tolvaptan appeared very safe, with minimal side effects. There was no increased incidence of electrolyte abnormalities or renal failure with extended tolvaptan therapy. Common side effects included dry mouth, thirst, and urinary frequency but these were not statistically significant compared with placebo.
In the EVEREST study, the long-term primary endpoints were all-cause mortality and the composite of cardiac-related death and hospitalization for CHF (Konstam et al 2007). Primary events were not different in the two groups after a mean follow-up time of 9.9 months. Again an increase in serum sodium levels was noted in patients treated with tolvaptan, and this increase was maintained through 40 weeks of therapy. The authors concluded that tolvaptan had no longterm effect on CHF morbidity or mortality.

In the third study, Udelson et al (2005) performed a randomized, double-blind, placebo-controlled study of 240 patients with $\mathrm{CHF}$ on standard therapy randomized to tolvaptan $30 \mathrm{mg}$ versus placebo for 52 weeks. In addition to clinical outcomes, they measured LVEDV by radionuclide ventriculography and the plasma levels of AVP, brain natriuretic peptide, norepinephrine, and renin at weeks 28 and 52. Progressive left ventricular dilatation occurs in the natural course of heart failure, a process that has been slowed, or possibly reversed, by ACE-I/ARB and beta-blocker therapy. Based on previous studies, it was unclear how tolvaptan would affect ongoing LV dilatation. On one hand, Gheorghiade's earlier studies suggested a survival benefit for patients receiving tolvaptan with severe congestive symptoms (Gheorghiade et al 2004). On the other, the question of unopposed $\mathrm{V}_{1 \mathrm{~A}}$ activity and increased AVP levels as a result of $\mathrm{V}_{2}$ blockade was concerning as excessive $\mathrm{V}_{1 \mathrm{~A}}$ receptor activity may contribute to cardiac myocyte hypertrophy and fibrosis (Costello-Boerrigter et al 2005). This could then lead to further dilatation of the ventricles in patients with CHF. Udelson et al (2005) found that there was no change in LVEDV in the placebo group and a non-significant decrease $\left(-1.8 \pm 10.7 \mathrm{~mL} / \mathrm{m}^{2}\right)$ in tolvaptan-treated patients at the end of 52 weeks $(\mathrm{p}=0.21$ compared with baseline LVEDV). AVP levels were significantly elevated in the tolvaptan-treated

Table 2 Long-term effects of tolvaptan on congestive heart failure

\begin{tabular}{|c|c|c|c|c|}
\hline Study & Event & Tolvaptan 30 mg & Placebo & p value \\
\hline \multirow[t]{3}{*}{ ACTIV in CHF } & Death & $13 / 239(5.4 \%)$ & $7 / 80(8.7 \%)$ & 0.18 \\
\hline & Rehospitalization & $44 / 239(18.4 \%)$ & | $4 / 80$ (I7.5\%) & $>0.99$ \\
\hline & Worsening CHF & $64 / 239$ (26.7\%) & $22 / 80(27.5 \%)$ & 0.88 \\
\hline \multirow[t]{2}{*}{ EVEREST } & All-cause mortality & $537 / 2063(25.9 \%)$ & $543 / 2055(26.3 \%)$ & 0.68 \\
\hline & $\begin{array}{l}\text { CV death or hospitalization } \\
\text { for } \mathrm{CHF}\end{array}$ & $87 I / 2063$ (42.0\%) & $829 / 2055$ (40.2\%) & 0.55 \\
\hline Effect of tolvaptan & Death & $6 / 120(5 \%)$ & I I/I20 (9\%) & 0.03 \\
\hline \multirow[t]{2}{*}{ on LV dilation } & CHF-related hospitalizations & $21 / 120(18 \%)$ & $34 / 120(28 \%)$ & \\
\hline & Post-LVEDI & $1.78 \pm 10.7$ & $0.04 \pm 10.0$ & 0.21 \\
\hline
\end{tabular}

Duration: ACTIV in CHF study: 60 days; EVEREST study: 9.9 months; and Udelson's Effect of Tolvaptan on LV Dilation and Function: 52 weeks. Abbreviations: CHF, congestive heart failure; CV, cardiovascular; LVEDVI, left ventricular end diastolic volume index. 
arm, but no other differences in neurohormones were noted. During the investigation, there were 6 deaths and 21 heart failure-related hospitalizations in the tolvaptan group versus 11 deaths and 34 hospitalizations in the placebo group. There was a significant advantage of tolvaptan on mortality and CHF hospitalization in a time-to-event analysis $(p<0.03$ by log-rank test). These secondary findings contradict those from the ACTIV in CHF and EVEREST trials, which showed no long-term beneficial effects of tolvaptan.

\section{Synergic effects between tolvaptan and diuretics}

In the study of Shoaf et al (2007), tolvaptan did not demonstrate any synergy with furosemide or HCTZ in healthy subjects. It should be mentioned that healthy subjects do not have elevated AVP levels or an activated renin-angiotensin-aldosterone system, therefore, the additive effects are likely to be limited. In patients with CHF or cirrhosis of the liver, these hormones are highly elevated as a compensatory mechanism for arterial underfilling (Schrier 2007). Diuretics were used in $>90 \%$ of patients in the previously described CHF studies (Gheorghiade et al 2003, 2004, 2007; Konstam et al 2007). Clearly the addition of tolvaptan to diuretics in treatment arms caused an increase in urine output compared with placebo plus diuretics. To further confirm the synergic effect, comparisons between tolvaptan alone versus tolvaptan plus diuretics in patients with CHF or cirrhosis are needed.

\section{Choice of vaptans for cirrhosis and congestive heart failure}

$\mathrm{V}_{1 \mathrm{~A}}+\mathrm{V}_{2}$ receptor versus $\mathrm{V}_{2}$ blockade alone in the setting of $\mathrm{CHF}$ and cirrhosis is another clinical question that needs further clarification. Due to $\mathrm{V}_{1 \mathrm{~A}}$ blockade, conivaptan tends to lower systolic and diastolic blood pressures (not statistically significant) (Ghali et al 2006). $\mathrm{V}_{1 \mathrm{~A}}$ receptors are abundant in the splanchnic circulation. In cirrhosis, a disease process characterized by pathologic vasodilation of this circulation, $\mathrm{V}_{1 \mathrm{~A}}$ blockade would be harmful. Theoretically, $\mathrm{V}_{2}$ receptor specific antagonists such as tolvaptan would be a better choice than conivaptan for treating cirrhosis-related hyponatremia. As for CHF, non-selective AVP antagonists may have advantages (Goldsmith 2007). Long-term use of tolvaptan is associated with an increase in endogenous AVP levels (Udelson et al 2005). This could potentially lead to unopposed $\mathrm{V}_{1 \mathrm{~A}}$ activation and an increase in afterload. Animal models have shown that myocardial cells contain $\mathrm{V}_{1 \mathrm{~A}}$ receptors and activation could trigger myocyte fibrosis
(Costello-Boerrigter et al 2005). Per the study by Udelson et al (2005), there did not appear to be differences in ventricular structure after 1 year of therapy with tolvaptan. This argues against significant myocyte fibrosis with unopposed $\mathrm{V}_{1 \mathrm{~A}}$ activity. Conivaptan may be a better choice in $\mathrm{CHF}$, but unfortunately the oral preparation has shown dramatic CYP3A4 inhibition, drug-drug interaction, and is not being developed by drug companies as a result (Ghali et al 2006). Clearly, trials that investigate and advantage of $\mathrm{V}_{1 \mathrm{~A}}+\mathrm{V}_{2}$ receptor versus $\mathrm{V}_{2}$ blockade in CHF are needed.

\section{Drug-drug interactions}

Tolvaptan is metabolized by the CYP3A4 system and therefore levels of the drug could be elevated with coadministration of P450 inhibitors such as ketoconazole and amiodarone (Shoaf et al 2005). Although $\mathrm{V}_{2}$ receptor antagonists have less CYP3A4 interaction in comparison to conivaptan, the maximal plasma concentration $\left(\mathrm{C}_{\max }\right)$ was increased by $248 \%$, and AUC (area under the plasma concentration versus time curve) $440 \%$ when tolvaptan was given along with ketoconazole (unpublished data). As many CHF patients that could benefit from tolvaptan are also taking amiodarone, this is an important drug interaction to investigate. Shoaf et al (2005) examined the question of interaction between tolvaptan and amiodarone in 2005. Patients maintained on oral amiodarone $200 \mathrm{mg}$ daily for $>10$ months were given subsequent doses of tolvaptan (30 mg day 3, $90 \mathrm{mg}$ day 4) while amiodarone and its metabolite desethylamoidarone disposition (DEA) levels were monitored. There was no significant change in these plasma markers for pre- versus post-tolvaptan dosing days. Tolvaptan concentrations were also measured and compared with previously gathered levels in CHF patients. Again no significant difference was noted in patients simultaneously receiving amiodarone. However, more studies are needed to determine whether long-term treatment with tolvaptan would have clinically significant effects due to drug drug-interaction.

\section{Conclusions}

Vasopressin receptor antagonists have clearly been the most exciting medications to come forth in the nephrology field for some time. Tolvaptan shows great promise in treating patients with hyponatremia. Despite minimal risk for overcorrection of sodium in the first 24 hours, it appears to be quite safe. Increased thirst and dry mouth are common, but were not severe enough for patients to stop therapy in trials. With non-compliance a huge obstacle in fluid restriction therapy, tolvaptan appears a better option. Patients with CHF show 
increased urine output and weight loss with tolvaptan. In acute decompensated heart failure tolvaptan will likely be very helpful in ameliorating symptoms and reducing length of hospital stay. However, long-term improvement in CHF morbidity and mortality has not been proved. Once approved by the FDA, tolvaptan is likely to have significant impact on clinical management of hyponatremia regardless of cause.

\section{Disclosures}

The authors have no conflicts of interest to disclose.

\section{References}

Costello-Boerrigter LC, Smith WB, Boerrigter G, et al. 2005. Vasopressin2-receptor antagonism augments water excretion without changes in renal hemodynamics or sodium and potassium excretion in human heart failure. Am J Physiol Renal Physiol, 290:F273-8.

Ghali JK, Koren MJ, Taylor JR, et al. 2006. Efficacy and safety of oral conivaptan: a V1A/V2 vasopressin receptor antagonist, assessed in a randomized, placebo-controlled trial in patients with euvolemic or hypervolemic hyponatremia. J Clin Endocrinol Metab, 91:2145-52.

Gheorghaide M, Gattis WA, O'Connor CM, et al. 2004. Effects of tolvaptan, a vasopressin antagonist, in patients hospitalized with worsening heart failure: a randomized controlled trial. JAMA, 291:1963-71.

Gheorghiade M, Gottlieb SS, Udelson JE, et al. 2006. Vasopressin $\mathrm{v}(2)$ receptor blockade with tolvaptan versus fluid restriction in the treatement of hyponatremia. Am J Cardiol, 97:1064-7.

Gheorghiade M, Konstam MA, Burnett JC Jr, et al. 2007. Short-term clinical effects of tolvaptan, an oral vasopressin antagonist, in patients hospitalized for heart failure: the EVEREST clinical status trials. JAMA, 297:1332-43.
Gheorghaide M, Niazi I, Ouyang J, et al. 2003. Vasopressin V2-receptor blockage with tolvaptan in patients with chronic heart failure: results from a double-blind, randomized trial. Circulation, 107:2690-6.

Goldsmith SR. 2007. Is there a cardiovascular rational for the use of combined V1A/V2 receptor antagonists? Am J Med, 119(Suppl 7A):S93-6.

Goldsmith SR, Francis GS, Cowley AW Jr, et al. 1983. Increased plasma arginine vasopressin levels in patients with congestive heart failure. J Am Coll Cardiol, 1:1385-90.

Konstam MA, Gheorgaide M, Burnett JC Jr, et al. 2007. Effects of oral tolvaptan in patients hospitalized for worsening heart failure: the EVEREST outcome trial. JAMA, 297:1319-31.

Lee DS, Austin PC, Rouleau JL, et al. 2003. Predicting mortality among patients hospitalized for heart failure: derivation and validation of a clinical model. JAMA, 290:2581-7.

Lien YH, Shapiro JI. 2007. Hyponatremia: clinical diagnosis and management. Am J Med, 120:653-8.

Schrier RW. 2007. Water and sodium retention in edematous disorders: role of vasopressin and aldosterone. Am J Med, 119(Suppl 7A):S47-53.

Schrier RW, Gross P, Gheorghiade M, et al. 2006. Tolvaptan, a selective oral vasopressin V2-receptor antagonist, for hyponatremia. $N$ Engl $J$ Med, 355:2099-112.

Shoaf SE, Elizari MV, Wang Z, et al. 2005. Tolvaptan administration does not affect steady state amiodarone concentrations in patients with cardiac arrhythmias. J Cardiovasc Pharmacol Ther, 10:165-71.

Shoaf SE, Graumer SL, Bricmont P, et al. 2007. Pharmacokinetic and harmacodynamic interaction between tolvaptan, an ono-peptide AV antagonist and furosemide or hydrochlorothiazide. J Cardiovasc Pharmacol, 50:213-2.

Udelson JE, McGrew FA, Flores E, et al. 2005. Multicenter, randomized, double-blind, placebo-controlled study on the effect of oral tolvaptan on left ventricular dilation and function inpatient with heart failure and systolic dysfunction. J Am Coll Cardiol, 49:2151-9. 
